Homology, Homotopy and Applications, vol. 8(2), 2006, pp.105-114

\title{
THE ETA INVARIANT AND THE "TWISTED" CONNECTIVE $K$-THEORY OF THE CLASSIFYING SPACE FOR CYCLIC 2-GROUPS
}

\author{
EGIDIO BARRERA-YANEZ
}

(communicated by Jonathan Rosenberg)

\begin{abstract}
Let $\ell=2^{\nu} \geqslant 2$. We use the eta invariant to study the "twisted" connective real $K$-theory groups $k o_{m}\left(B \mathbb{Z}_{\ell}, \xi_{1}\right)$ of the classifying space $B \mathbb{Z}_{\ell}$ for the cyclic group $\mathbb{Z}_{\ell}$.
\end{abstract}

\section{Introduction}

Atiyah [1] expressed the complex $K$-theory of the classifying space of $\mathbb{Z}_{\ell}$ in terms of the complex representation ring of $\mathbb{Z}_{\ell}$. Looking for a "geometric" construction of Elliptic homology, Kreck and Stolz [11] gave a geometric characterization of connective real $K$-theory. Stolz used this characterization to study when a simply connected manifold admits a metric with positive scalar curvature, see [12] for details. Botvinnik and Gilkey [6] and Botvinnik, Gilkey, and Stolz [7] studied when a manifold with non-trivial fundamental group admits a metric with positive scalar curvature. They defined a "twisted" geometric version of connective real $K$-theory. In this paper we will express the "twisted" connective real $K$-theory of the classifying space $\mathbb{Z}_{\ell}$ in terms of the complex representation ring of $\mathbb{Z}_{\ell}$. Instead of using topological methods as Atiyah did, we shall use analytical methods; our fundamental tool is the eta invariant.

Let $\mathbb{Z}_{\ell}:=\left\{\lambda \in \mathbb{C}: \lambda^{\ell}=1\right\}$ be the cyclic group of order $\ell=2^{\nu} \geqslant 2$. Let $\rho_{s}(\lambda)=\lambda^{s}$ define an irreducible linear representation of $\mathbb{Z}_{\ell}$. The $\rho_{s}$ parametrize the irreducible representations of $\mathbb{Z}_{\ell}$. Let $\xi_{1}$ be the underlying real 2 plane bundle of the complex line bundle defined by the representation $\rho_{1}$. Let $D\left(\xi_{1}\right), S\left(\xi_{1}\right)$ be the disk bundle and respectively sphere bundle with respect to some fiber metric on $\xi_{1}$. Let $T\left(\xi_{1}\right)=$ $D\left(\xi_{1}\right) / S\left(\xi_{1}\right)$ be the Thom space associated with $\xi_{1}$. We use the Thom-Pontryagin construction to define the twisted equivariant spin bordism groups and twisted connective real $K$-theory groups by:

$$
\begin{array}{r}
M \operatorname{Spin}_{m}\left(B \mathbb{Z}_{\ell}, \xi_{1}\right):={\widetilde{M \operatorname{Spin}_{m+2}}}\left(T\left(\xi_{1}\right)\right) \\
k o_{m}\left(B \mathbb{Z}_{\ell}, \xi_{1}\right):=\widetilde{k o}_{m+2}\left(T\left(\xi_{1}\right)\right) .
\end{array}
$$

$\dagger$ The author passed away on August 19, 2004.

Received January 12, 2004; published on August 9, 2006.

2000 Mathematics Subject Classification: 55N15, 58G12.

Key words and phrases: connective $K$-theory, eta invariant.

Copyright (C) 2006, International Press. Permission to copy for private use granted. 
We refer to $[\mathbf{6}, \mathbf{7}]$ for further details. Notice that if we replace $\xi_{1}$ by the trivial line bundle $\xi_{0}$, then we get the standard untwisted equivariant bordism and real connective $K$-theory groups. In Section 2.2, we shall give a more geometric definition of these groups.

The calculations of $[\mathbf{6}]$ using the Adams Spectral Sequence show that the groups $k o_{8 k+i}\left(B \mathbb{Z}_{\ell}, \xi_{1}\right)$ have finite order for $i=1,3,5,7$ and that $k o_{m}\left(B \mathbb{Z}_{\ell}, \xi_{1}\right)=0$ otherwise. The additive structure of these groups has not been determined previously. We note that the additive structures of the groups $k o_{*}\left(B \mathbb{Z}_{\ell}\right)$ were studied in [5].

We can now state the main result of this paper. If $\tau: \mathbb{Z}_{\ell} \rightarrow U(2 k)$ is a fixed point free representation, we denote the associated lens space by

$$
L^{4 k-1}(\ell ; \tau):=S^{4 k-1} / \tau\left(\mathbb{Z}_{\ell}\right) .
$$

Let $R U_{0}\left(\mathbb{Z}_{\ell}\right)$ be the augmentation ideal of the unitary group representation ring $R U\left(\mathbb{Z}_{\ell}\right)$. Let $\widetilde{K S p}$ and $\widetilde{K U}$ be the reduced symplectic and complex $K$-theory groups. Let

$$
\mathcal{I}=\left\{\rho \in R U_{0}\left(\mathbb{Z}_{\ell}\right): \rho(\bar{\lambda})=-\rho(\lambda)\right\}
$$

Theorem 1.1. Let $k \geqslant 1$.

1. We have that $k o_{4 k+1}\left(B \mathbb{Z}_{\ell}, \xi_{1}\right) \approx \widetilde{K S p}\left(L^{4 k+5}(\ell, \tau)\right)$ for any suitable $\tau$.

2. We have that $k_{4 k-1}\left(B \mathbb{Z}_{\ell}, \xi_{1}\right) \approx \mathcal{I} /\left(\mathcal{I} \cap R U_{0}\left(\mathbb{Z}_{\ell}\right)^{2 k+2}\right)$.

Here is a brief guide to this paper. In Section 2.3, we review the properties concerning the eta invariant which we shall need. In Section 3, we prove the main Theorem.

\section{Acknowledgements}

It is a pleasant task to thank Professors Peter B. Gilkey, Jose Luis Cisneros Molina, and Santos Asin Lares for valuable and stimulating discussions. We also wish to acknowledge the hospitality of The Abdus Salam International Centre for Theoretical Physics where much of this research was performed.

\section{The eta invariant and "twisted" connective real $K$-theory}

\subsection{Notational conventions}

If $\rho$ is a virtual unitary or symplectic representation, let $V_{\rho}$ be the associated virtual flat bundle and let $\left[V_{\rho}\right]$ be the corresponding element in $K$-theory. Let $M=$ $L^{4 k-1}(\ell ; \tau)$. The map $\rho \rightarrow\left[V_{\rho}\right]$ defines a surjective homomorphism from $R U_{0}\left(\mathbb{Z}_{\ell}\right)$ and $R S p_{0}\left(\mathbb{Z}_{\ell}\right)$ to $\widetilde{K U}(M)$ and $\widetilde{K S p}(M)$; see $[\mathbf{2 , 1 0}]$.

\subsection{A geometrical realization of connective $K$-theory and bordism}

The equivariant spin bordism groups $\operatorname{MSpin}_{m}\left(B \mathbb{Z}_{\ell}, \xi_{1}\right)$ can also be thought of as equivalence classes of triples $(M, f, s)$ where $M$ is a closed manifold of dimension $m$ which need not be connected, $f$ is a $\mathbb{Z}_{\ell}$ structure on $M$, and $s$ is a spin structure on $T(M) \oplus f^{*}\left(\xi_{1}\right)$; such a manifold $M$ admits a $\operatorname{spin}^{c}$ structure with determinant 
line bundle given by $\rho_{1}$, see $[\mathbf{4 , 6}]$ for details. We define the relation $(M, f, s) \sim 0$ in $M \operatorname{Spin}_{m}\left(B \mathbb{Z}_{\ell}, \xi_{1}\right)$ if there exists a compact manifold $N$ with boundary $M$ so that the structures $s$ and $f$ extend over $N$. Disjoint union defines the group structure; Cartesian product makes $M \operatorname{Spin}_{*}\left(B \mathbb{Z}_{\ell}, \xi_{1}\right)$ into a $M \operatorname{Spin}_{*}$ module. Let $T_{m}\left(B \mathbb{Z}_{\ell}, \xi_{1}\right)$ be the subgroup of $M \operatorname{Spin}_{m}\left(B \mathbb{Z}_{\ell}, \xi_{1}\right)$ which is generated by the total spaces of geometrical fiber bundles $p: E \rightarrow B$ with fiber quaternionic projective space $\mathbb{H} \mathbb{P}^{2}$; since $\mathbb{H} \mathbb{P}^{2}$ is simply connected, we can identify $\mathbb{Z}_{\ell}$ structures on the base with those on the total space. We refer to Stolz [13] for the proof of the following lemma; it is fundamental to our study since it expresses the "twisted" connective real $K$-theory groups of $B \mathbb{Z}_{\ell}$ geometrically.

Theorem 2.1. $k o_{m}\left(B \mathbb{Z}_{\ell}, \xi_{1}\right)=M \operatorname{Spin}_{m}\left(B \mathbb{Z}_{\ell}, \xi_{1}\right) / T_{m}\left(B \mathbb{Z}_{\ell}, \xi_{1}\right)$.

\subsection{The eta invariant}

Let $D=D(M, g, s)$ be the Dirac operator defined by the spin structure $s$ and a Riemannian metric $g$, and let $\rho$ be a complex representation of $\mathbb{Z}_{\ell}$. Let $D_{\rho}$ be the Dirac operator with coefficients in the flat vector bundle $V_{\rho}$ defined by $\rho$. Let $\left\{\lambda_{n}\right\}$ be the eigenvalues of $D_{\rho}$ where each eigenvalue is repeated according to its multiplicity. The function $\eta\left(D_{\rho}, z\right):=\sum_{\lambda_{n} \neq 0} \operatorname{sign}\left(\lambda_{n}\right)\left|\lambda_{n}\right|^{-z}$ converges to a holomorphic function for $\Re e(z) \gg 0$. This function has a meromorphic extension to $\mathbb{C}$. The value $z=0$ is a regular value and we define

$$
\eta(M, f, s)(\rho):=\frac{1}{2}\left(\left.\eta\left(D_{\rho}, z\right)\right|_{z=0}+\operatorname{dim} \operatorname{ker}\left(D_{\rho}\right)\right)
$$

as a measure of the spectral asymmetry of $D_{\rho}$. We refer to $[\mathbf{3}, \mathbf{9}]$ for details. The Atiyah-Patodi-Singer Index Theorem for manifolds with boundary [3] can be used to establish the following result. We refer to $[\mathbf{6}]$ (Thm 3.1), [7] for details:

Theorem 2.2. If $m=4 k+1$, let $\rho \in R U\left(\mathbb{Z}_{\ell}\right)$; if $m=4 k-1$, let $\rho \in R U_{0}\left(\mathbb{Z}_{\ell}\right)$. The map $M \rightarrow \eta(M, f, s)(\rho)$ extends to a homomorphism $\eta_{\rho}$ from $k o_{m}\left(B \mathbb{Z}_{\ell}, \xi_{1}\right)$ to $\mathbb{R} / \mathbb{Z}$.

\subsection{Lens spaces and lens space bundles}

Let $\vec{a}=\left(a_{1}, \ldots, a_{k}\right)$ be a collection of odd indices and let $\tau=\tau(\vec{a}):=\rho_{a_{1}} \oplus \ldots \oplus$ $\rho_{a_{k}}$ define a fixed point free representation from $\mathbb{Z}_{\ell}$ to $U(k)$. Let $L^{2 k-1}(\ell, \tau):=$ $S^{2 k-1} / \tau\left(\mathbb{Z}_{\ell}\right)$ be the associated lens space. Let $H^{\otimes 2} \oplus(k-1) 1$ be the Whitney sum of the tensor square of the complex Hopf line bundle with $(k-1)$ copies of the trivial complex line bundle over complex projective space $\mathbb{C P}^{1}$ which we identify with the sphere $S^{2}$. We let $\lambda \in S^{1}$ act by multiplication by $\lambda^{a_{\nu}}$ on the $\nu^{t h}$ summand. This action restricts to a fixed point free action of $\mathbb{Z}_{\ell}$ on the associated sphere bundle. Let

$$
X^{2 k+1}(\ell ; \tau):=S\left(H^{\otimes 2} \oplus(k-1) 1\right) / \tau\left(\mathbb{Z}_{\ell}\right)
$$

be the associated lens space bundle over $S^{2}$. We give $L^{2 k-1}(\ell, \tau)$ and $X^{2 k+1}(\ell ; \tau)$ the natural $\mathbb{Z}_{\ell}$ structures. The lens space $L^{2 k-1}(\ell ; \tau)$ and the lens space bundles $X^{2 k+1}(\ell ; \tau)$ admit a natural $\operatorname{spin}^{c}$ structure; see $[\mathbf{4}, \mathbf{6}, \mathbf{7}]$ for details. 


\subsection{Combinatorial formulas for the eta invariant}

Let $\tau=\tau(\vec{a})$. We define

1. If $k$ is even, let $\mathcal{F}_{L}(\vec{a} ; \lambda)=\lambda^{-|\vec{a}| / 2} \operatorname{det}(I-\tau(\vec{a})(\lambda))$.

2. If $k$ is odd, let $\mathcal{F}_{L}(\vec{a} ; \lambda)=\lambda^{-(|\vec{a}|+1) / 2} \operatorname{det}(I-\tau(\vec{a})(\lambda))$.

3. If $\lambda \neq 1$, let $\mathcal{G}_{L}(\vec{a} ; \lambda)=\mathcal{F}_{L}(\vec{a} ; \lambda)^{-1}$. If $\lambda=1$, let $\mathcal{G}_{L}(\vec{a} ; \lambda)=0$.

4. Let $\mathcal{G}_{X}(\vec{a} ; \lambda)=\left(1+\lambda^{a_{1}}\right)\left(1-\lambda^{a_{1}}\right)^{-1} \mathcal{G}_{L}(\vec{a} ; \lambda)$.

Let $\widetilde{\sum}_{\lambda}:=\sum_{\lambda \in \mathbb{Z}_{\ell}, \lambda \neq 1}$. The following combinatorial formulas follow from work of Donnelly $[\mathbf{8}]$ :

Theorem 2.3. 1. We have $\eta\left(L^{4 k-1}(\ell ; \tau)\right)(\rho)=\ell^{-1} \widetilde{\sum}_{\lambda} \operatorname{Tr}(\rho(\lambda)) \mathcal{G}_{L}(\tau)(\lambda)$.

2. We have $\eta\left(X^{4 k+1}(\ell ; \tau)\right)(\rho)=\ell^{-1} \widetilde{\sum}_{\lambda} \operatorname{Tr}(\rho(\lambda)) \mathcal{G}_{X}(\tau)(\lambda)$.

The eta invariant completely detects the odd dimensional "twisted" connective $K$-theory groups $k o_{m}\left(B \mathbb{Z}_{\ell}, \xi_{1}\right)$. We refer to $[\mathbf{6}]$ for the proof of the following result. Notice that these manifolds have positive scalar curvature, therefore $\hat{A}=0$.

Theorem 2.4. Let $M:=[(M, f, s)] \in k o_{m}\left(B \mathbb{Z}_{\ell}, \xi_{1}\right)$.

1. If $m \equiv 1 \bmod 8$, then $M=0$ if and only if $\eta(M)(\rho)=0$ in $\mathbb{R} / \mathbb{Z}$ for all $\rho \in$ $R U\left(\mathbb{Z}_{\ell}\right)$.

2. If $m \equiv 3 \bmod 8$, then $M=0$ if and only if $\eta(M)(\rho)=0$ in $\mathbb{R} / \mathbb{Z}$ for all $\rho \in$ $R U_{0}\left(\mathbb{Z}_{\ell}\right)$.

3. If $m \equiv 5 \bmod 8$, then $M=0$ if and only if $\eta(M)(\rho)=0$ in $\mathbb{R} / \mathbb{Z}$ for all $\rho \in$ $R U\left(\mathbb{Z}_{\ell}\right)$.

4. If $m \equiv 7 \bmod 8$, then $M=0$ if and only if $\eta(M)(\rho)=0$ in $\mathbb{R} / \mathbb{Z}$ for all $\rho \in$ $R U_{0}\left(\mathbb{Z}_{\ell}\right)$.

Let $c_{S p}: R S p\left(\mathbb{Z}_{\ell}\right) \rightarrow R U\left(\mathbb{Z}_{\ell}\right)$ be the natural injective homomorphism obtained by forgetting the symplectic structure to get a complex structure.

We refer to $[\mathbf{2}, \mathbf{9}, \mathbf{1 0}]$ for the proof of the following result.

Theorem 2.5. Let $M:=L^{2 k-1}(\ell ; \tau)$.

1. Let $\rho \in R U_{0}\left(\mathbb{Z}_{\ell}\right)$. The following conditions are equivalent:

(a) $\rho \in R U_{0}\left(\mathbb{Z}_{\ell}\right)^{k}$.

(b) $\eta(M)(\rho \tilde{\rho}) \in \mathbb{Z} \forall \tilde{\rho} \in R U_{0}\left(\mathbb{Z}_{\ell}\right)$.

(c) $\left[V_{\rho}\right]=0$ in $\widetilde{K U}(M)$.

2. Let $\gamma=c_{S p}(\rho)$ for $\rho \in R S p_{0}\left(\mathbb{Z}_{\ell}\right)$. If $2 k-1 \equiv 7 \bmod 8$, the following conditions are equivalent:

(a) $\gamma \in \psi^{k} c_{S p} R S p_{0}\left(\mathbb{Z}_{\ell}\right)$ where $\psi=\left(\rho_{0}-\rho_{1}\right)^{2} \rho_{-1}$.

(b) $\eta(M)(\gamma \tilde{\rho}) \in \mathbb{Z} \forall \tilde{\rho} \in R U_{0}\left(\mathbb{Z}_{\ell}\right)$ and $(\ell / 2) \eta(M)(\gamma) \in \mathbb{Z}$.

(c) $\left[V_{\rho}\right]=0$ in $\widetilde{K S p}(M)$. 
3. Let $\gamma=c_{S p}(\rho)$ for $\rho \in R S p_{0}\left(\mathbb{Z}_{\ell}\right)$. If $2 k-1 \equiv 3 \bmod 8$, the following conditions are equivalent:

(a) $\gamma \in R U_{0}\left(\mathbb{Z}_{\ell}\right)^{k}$.

(b) $\eta(M)(\gamma \tilde{\rho}) \in \mathbb{Z} \forall \tilde{\rho} \in R U_{0}\left(\mathbb{Z}_{\ell}\right)$.

(c) $\left[V_{\rho}\right]=0$ in $\widetilde{K S p}(M)$.

\section{The proof of the main theorem}

We consider the free Abelian group generated by the lens spaces $L^{4 k+1}(\ell ; \vec{a})$ and by the lens space bundles $X^{4 k-1}(\ell ; \vec{a})$; we give these manifolds the natural structures and omit these structures from the notation in the interests of notational simplicity. Define:

1. $\mathcal{B} L^{4 k+1}(\ell ; \cdot, 3):=3 L^{4 k+1}(\ell ; \cdot, 3)-L^{4 k+1}(\ell ; \cdot, 1)$.

2. $\mathcal{B} X^{4 k-1}(\ell ; \cdot, 3):=3 X^{4 k-1}(\ell ; \cdot, 3)-X^{4 k-1}(\ell ; \cdot, 1)$.

This defines $\mathcal{B}$ on any lens space and lens space bundle. We extend $\mathcal{B}$ to the free Abelian group generated by these manifolds. We define $M_{m, j}^{L}$ for $2 j-1 \leqslant m$. When considering the lens space bundles, we assume the index " 3 " in question is not the first index. Thus we define $M_{m, j}^{X}$ for $2 j-1 \leqslant m-4$. The eta invariant is additive with respect to direct sums and extends to this setting.

1. $M_{4 k+1, j}^{L}:=\mathcal{B}^{j} L^{4 k+1}(\ell ; 3, \ldots, 3)$.

2. $M_{4 k-1, j}^{X}:=\mathcal{B}^{j} X^{4 k-1}(\ell ; 1,3, \ldots, 3)$.

Theorem 3.1. We have

$$
\begin{array}{r}
k o_{4 k+1}\left(B \mathbb{Z}_{\ell}, \xi_{1}\right)=\operatorname{span}\left\{M_{4 k+1, j}^{L}: 0 \leqslant j \leqslant 2 k+1\right\}, \text { and } \\
k o_{4 k-1}\left(B \mathbb{Z}_{\ell} ; \xi_{1}\right)=\operatorname{span}\left\{M_{4 k-1, j}^{X}: 0 \leqslant j \leqslant 2 k-2\right\}
\end{array}
$$

Proof. We prove this theorem with the following lemmas.

We refer to [6] (Lemma 4.2) for the proof of the following result.

Lemma 3.2. Let $\widetilde{\sum}_{\lambda}:=\sum_{\lambda \in \mathbb{Z}_{\ell}, \lambda \neq 1}$.

1. If $k$ is even, then $L^{2 k-1}(\ell ; \vec{a})$ and $X^{2 k+1}(\ell ; \vec{a})$ admit spin structures.

2. If $k$ is odd, then $L^{2 k-1}(\ell ; \vec{a})$ and $X^{2 k+1}(\ell ; \vec{a})$ have spin ${ }^{c}$ structures with determinant line bundle given by $\rho_{1}$.

3. We have $\eta\left(L^{2 k-1}(\ell ; \vec{a})\right)(\rho)=\ell^{-1} \widetilde{\sum}_{\lambda} \operatorname{Tr}(\rho) \mathcal{G}_{L}(\vec{a} ; \lambda) \in \mathbb{Q}$.

4. We have $\eta\left(X^{2 k+1}(\ell ; \vec{a})\right)(\rho)=\ell^{-1} \widetilde{\sum}_{\lambda} \operatorname{Tr}(\rho) \mathcal{G}_{X}(\vec{a} ; \lambda) \in \mathbb{Q}$.

We have the following integrality theorem; we refer to [6] (Lemma 4.2) for the proof.

Lemma 3.3. Let $\rho \in R U_{0}\left(\mathbb{Z}_{\ell}\right)^{j}$. Let $m<2 j+1$. Then

$$
\eta\left(L^{m}(\ell ; \cdot)\right)(\rho) \in \mathbb{Z} \text { and } \eta\left(X^{m}(\ell ; \cdot)\right)(\rho) \in \mathbb{Z} .
$$


Lemma 3.4. Let $\rho \in R U\left(\mathbb{Z}_{\ell}\right)$ and let $\psi:=\left(\rho_{0}-\rho_{1}\right)^{2} \rho_{-1}$.

1. $\eta(\mathcal{B} M)(\rho)=\eta(M)(\psi \rho)$ for $M$ a lens space or suitable lens space bundle.

2. $\eta\left(M_{m, j}^{L}\right)(\rho)=\eta\left(M_{m, 0}^{L}\right)\left(\psi^{j} \rho\right)$ and $\eta\left(M_{m, j}^{X}\right)(\rho)=\eta\left(M_{m, 0}^{X}\right)\left(\psi^{j} \rho\right)$

Proof. We see that

$$
\mathcal{G}_{L}(\vec{a}, 1 ; \lambda)-3 \mathcal{G}_{L}(\vec{a}, 3 ; \lambda)=\psi(\lambda) \mathcal{G}_{L}(\vec{a}, 3 ; \lambda) .
$$

Consequently,

$$
\eta\left(\mathcal{B} L^{2 k+1}(\ell ; \vec{a}, 3)\right)(\rho)=\eta\left(L^{2 k+1}(\ell ; \vec{a}, 3)\right)(\psi \rho)
$$

and assertions concerning lens spaces follow. Similarly,

$$
\eta\left(\mathcal{B} X^{2 k+3}(\ell ; \vec{a}, 3)\right)(\rho)=\eta\left(X^{2 k+3}(\ell ; \vec{a}, 3)\right)(\psi \rho)
$$

provided that the index " 3 " is not the first index; the first index plays a distinguished role in the definition of $\mathcal{G}_{X}$.

Lemma 3.5. Let $\alpha:=\rho_{-3}\left(\rho_{0}-\rho_{3}\right)^{2} \in R U_{0}\left(\mathbb{Z}_{\ell}\right)^{2}$.

1. $\eta\left(M_{m, j}^{L}\right)(\alpha \rho)=\eta\left(M_{m-4, j}^{L}\right)(\rho)$.

2. $\eta\left(M_{m, j}^{X}\right)(\alpha \rho)=\eta\left(M_{m-4, j}^{X}\right)(\rho)$.

3. $\eta\left(M_{5,0}^{L}\right)\left(\alpha \rho_{-2}\right)=(\ell-1) / 2 \ell$.

4. $\eta\left(M_{5,0}^{X}\right)\left(\alpha\left(\rho_{0}-\rho_{3}\right) \rho_{-2}\right)=(\ell-2) / \ell$.

5. If $\rho \in R U\left(\mathbb{Z}_{\ell}\right)$, then $\eta\left(M_{4 k+1, k}^{L}\right)(\alpha \rho) \in \mathbb{Z}$. Furthermore there exists $\gamma_{4 k+1}^{L}$ so that $\eta\left(M_{4 k+1, k}^{L}\right)(\alpha \rho)\left(\gamma_{4 k+1}^{L}\right)=(\ell-1) / 2 \ell$.

6. If $\rho \in R U\left(\mathbb{Z}_{\ell}\right)$, then $\eta\left(M_{4 k+1, k}^{X}\right)(\alpha \rho) \in \mathbb{Z}$. Furthermore there exists $\gamma_{4 k+1}^{X}$ so that $\eta\left(M_{4 k+1, k}^{L}\right)(\alpha \rho)\left(\gamma_{4 k+1}^{X}\right)=(\ell-2) / \ell$.

Proof. Since $\mathcal{F}(\vec{a}, 3,3 ; \lambda)=\alpha(\lambda) \mathcal{F}(\vec{a} ; \lambda)$,

$$
\begin{gathered}
\eta\left(L^{m+4}(\ell ; \vec{a}, 3,3)\right)(\alpha \rho)=\eta\left(L^{m}(\ell ; \vec{a})\right)(\rho) \\
\eta\left(X^{m+4}(\ell ; \vec{a}, 3,3)\right)(\alpha \rho)=\eta\left(X^{m}(\ell ; \vec{a})\right)(\rho) .
\end{gathered}
$$

The first two assertions now follow. We prove the second two assertions by computing:

$$
\begin{aligned}
\eta\left(M_{5,0}^{L}\right)\left(\alpha \rho_{-2}\right) & =\ell^{-1} \widetilde{\sum}_{\lambda}\left(1-\lambda^{3}\right)^{-1} \\
& =(2 \ell)^{-1}{\widetilde{\sum_{\lambda}}}_{\lambda}\left((1-\lambda)^{-1}+(1-\bar{\lambda})^{-1}\right) \\
& =(2 \ell)^{-1} \widetilde{\sum}_{\lambda} 1=(\ell-1) /(2 \ell)
\end{aligned}
$$

and

$$
\eta\left(M_{5,0}^{X}\right)\left(\alpha\left(\rho_{0}-\rho_{3}\right) \rho_{-2}\right)=\ell^{-1} \widetilde{\sum}_{\lambda}\left(1+\lambda^{3}\right)=\ell^{-1} \widetilde{\sum}_{\lambda}(1+\lambda)=(\ell-2) / \ell .
$$

We complete the proof by establishing the final two assertions. We use Lemma 3.4 to compute 
Homology, Homotopy and Applications, vol. 8(2), 2006

$$
\begin{array}{r}
\eta\left(M_{4 k+1, k}^{L}\right)(\alpha \rho)=\eta\left(M_{4 k+1,0}^{L}\right)\left(\alpha \psi^{k} \rho\right), \text { and } \\
\eta\left(M_{4 k+1, k}^{X}\right)(\alpha \rho)=\eta\left(M_{4 k+1,0}^{X}\right)\left(\alpha \psi^{k} \rho\right) .
\end{array}
$$

Then $\rho \alpha \psi^{k} \in R U_{0}(\mathbb{Z} \ell)^{2 k+2}$. Since $\operatorname{dim}\left(M_{4 k+1,0}^{L}\right)=\operatorname{dim}\left(M_{4 k+1,0}^{X}\right)=2(2 k+1)-1$, these eta invariants take values in $\mathbb{Z}$ by Lemma 3.3. Similarly, we compute:

$$
\begin{array}{r}
\eta\left(M_{4 k+1, k}^{L}\right)\left(\gamma_{k, L}\right)=\eta\left(M_{m, 0}^{L}\right)\left(\gamma_{k, L} \psi^{k}\right), \text { and } \\
\eta\left(M_{4 k+1, k}^{X}\right)\left(\gamma_{k, X}\right)=\eta\left(M_{m, 0}^{X}\right)\left(\gamma_{k, X} \psi^{k}\right) .
\end{array}
$$

We have $\psi^{k} R\left(\mathbb{Z}_{\ell}\right)=\alpha^{k} R\left(\mathbb{Z}_{\ell}\right)$. Thus we may choose $\gamma_{k, L}$ so that $\gamma_{k, L} \psi^{k}=\alpha^{k} \rho_{-2}$; let $\gamma_{k, X}=\gamma_{k, L}\left(\rho_{0}-\rho_{3}\right)$. Then

$$
\begin{array}{r}
\eta\left(M_{m, 0}^{L}\right)\left(\gamma_{k, L} \psi^{k}\right)=\eta\left(M_{m, 0}^{L}\right)\left(\alpha^{k} \rho_{-2}\right)=\eta\left(M_{5,0}^{L}\right)\left(\alpha \rho_{-2}\right) \\
=(\ell-1) / 2 \ell \\
\eta\left(M_{m, 0}^{X}\right)\left(\gamma_{k, X} \psi^{k}\right)=\eta\left(M_{m, 0}^{L}\right)\left(\alpha^{k}\left(\rho_{0}-\rho_{3}\right) \rho_{-2}\right) \\
=\eta\left(M_{5,0}^{L}\right)\left(\alpha\left(\rho_{0}-\rho_{3}\right) \rho_{-2}\right)=(\ell-2) / \ell .
\end{array}
$$

Let $k \geqslant 0$. We define

1. $\mathcal{M}_{4 k+1}^{L}(\ell):=\operatorname{span}_{0 \leqslant j \leqslant 2 k+1}\left\{M_{4 k+1, j}^{L}\right\} \subset k o_{4 k+1}\left(B \mathbb{Z}_{\ell}, \xi_{1}\right)$.

2. $\mathcal{M}_{4 k-1}^{X}(\ell):=\operatorname{span}_{0 \leqslant j \leqslant 2 k-2}\left\{M_{4 k-1, j}^{X}\right\} \subset k o_{4 k-1}\left(B \mathbb{Z}_{\ell}, \xi_{1}\right)$.

The Pontryagin dual $A^{*}$ of an Abelian group $A$ is the group of homomorphisms to $\mathbb{R} / \mathbb{Z}$. Thus, for example, $\mathbb{Z} / \ell \mathbb{Z}$ is the Pontryagin dual of $\mathbb{Z}_{\ell}$. Let $\eta^{*}(M)$ be the homomorphism which sends $\rho$ to $\eta(M)(\rho)$. By Theorem 2.5, the eta invariant extends to connective $K$-theory:

$$
\eta^{*}: k o_{4 k+1}\left(B \mathbb{Z}_{\ell}, \xi_{1}\right) \rightarrow R U\left(\mathbb{Z}_{\ell}\right)^{*} \text {, and } \eta^{*}: k o_{4 k-1}\left(B \mathbb{Z}_{\ell}, \xi_{1}\right) \rightarrow R U_{0}\left(\mathbb{Z}_{\ell}\right)^{*} \text {. }
$$

The homomorphism which sends $\rho$ to $\alpha \rho$ defines a dual map $\alpha^{*}$ from $R U\left(\mathbb{Z}_{\ell}\right)^{*}$ to $R U\left(\mathbb{Z}_{\ell}\right)^{*}$.

Lemma 3.6. Let $k \geqslant 0$ and assume $\ell \geqslant 4$.

1. $\left|\eta^{*} \mathcal{M}_{4 k+5}^{L}(\ell)\right| \geqslant(2 \ell)^{k+2}$.

2. $k o_{4 k+5}\left(B \mathbb{Z}_{\ell}, \xi_{1}\right)=\mathcal{M}_{4 k+5}^{L}(\ell)$.

Proof. It is immediate that

$$
\left|\eta^{*} \mathcal{M}_{m}^{L}(\ell)\right| \geqslant\left|\alpha^{*} \eta^{*} \mathcal{M}_{m}^{L}(\ell)\right| \cdot\left|\operatorname{ker} \alpha^{*} \cap \eta^{*} \mathcal{M}_{m}^{L}(\ell)\right|
$$

We use Lemma 3.5 to see that

$$
\begin{array}{r}
\left|\alpha^{*} \eta^{*} \mathcal{M}_{m}^{L}(\ell)\right| \geqslant\left|\eta^{*} \mathcal{M}_{m-4}^{L}(\ell)\right|, \\
\left|\alpha^{*} \eta^{*} \mathcal{M}_{5}^{L}(\ell)\right| \geqslant 2 \ell \\
\left|\operatorname{ker} \alpha^{*} \cap \eta^{*} \mathcal{M}_{m}^{L}(\ell)\right| \geqslant 2 \ell .
\end{array}
$$

This proves the first assertion and gives a lower bound for $k o_{m}\left(B \mathbb{Z}_{\ell}, \xi_{1}\right)$ if $m \equiv 1$ $\bmod 4$. 
The following estimates were established in Botvinnik and Gilkey [6].

1. $\left|k o_{8 k+1}\left(\mathbb{Z}_{\ell}, \xi_{1}\right)\right|=(2 \ell)^{2 k+1}$.

2. $\left|k o_{8 k+3}\left(\mathbb{Z}_{\ell}, \xi_{1}\right)\right|=(\ell / 2)^{2 k+1}$.

3. $\left|k o_{8 k+5}\left(\mathbb{Z}_{\ell}, \xi_{1}\right)\right|=(2 \ell)^{2 k+2}$.

4. $\left|k o_{8 k+7}\left(\mathbb{Z}_{\ell}, \xi_{1}\right)\right|=(\ell / 2)^{2 k+2}$.

The final assertion follows from these estimates.

Again, we begin our discussion with a technical Lemma.

Lemma 3.7. 1. $\eta\left(M_{3,0}^{L}\right)\left(\left(\rho_{0}-\rho_{3}\right) \rho_{-2}\right)=(\ell-1) / 2 \ell$.

2. $\eta\left(M_{3,0}^{X}\right)\left(\left(\rho_{0}-\rho_{3}\right)^{2} \rho_{-2}\right)=(\ell-2) / \ell$.

Proof. We prove the first assertion by computing:

$$
\begin{aligned}
\eta\left(M_{3,0}^{L}\right)\left(\left(\rho_{0}-\rho_{3}\right) \rho_{-2}\right) & =\ell^{-1} \widetilde{\sum}_{\lambda}\left(1-\lambda^{3}\right)^{-1} \\
& =(2 \ell)^{-1} \widetilde{\Sigma}_{\lambda}\left((1-\lambda)^{-1}+(1-\bar{\lambda})^{-1}\right) \\
& =(2 \ell)^{-1} \widetilde{\Sigma}_{\lambda} 1=(\ell-1) /(2 \ell) .
\end{aligned}
$$

and

$$
\eta\left(M_{3,0}^{X}\right)\left(\left(\rho_{0}-\rho_{3}\right)^{2} \rho_{-2}\right)=\ell^{-1} \widetilde{\sum}_{\lambda}\left(1+\lambda^{3}\right)=\ell^{-1} \widetilde{\sum}_{\lambda}(1+\lambda)=(\ell-2) / \ell .
$$

Lemma 3.8. If $k \geqslant 0$ and if $\ell \geqslant 4$, then
1. $\mid \eta^{*}\left(\mathcal{M}_{4 k+3}^{X}(\ell) \mid \geqslant(\ell / 2)^{k+1}\right.$.
2. ${ } o_{4 k+3}\left(B \mathbb{Z}_{\ell}, \xi_{1}\right)=\mathcal{M}_{4 k+3}^{X}(\ell)$.

Proof. The first assertion follows from Lemma 3.7 if $k=0$, so we assume $k \geqslant 1$ henceforth. Let $\delta=\left(\rho_{0}-\rho_{3}\right) \rho_{-2}$ and let $m=4 k+3$. Then,

$$
\eta\left(M_{m, j}^{X}(\ell)\right)(\delta \rho)=\eta\left(M_{m-2, j}^{X}(\ell)\right)(\rho) .
$$

Thus,

$$
\eta^{*} \mathcal{M}_{4 k+1}^{X} \subset \delta^{*} \eta^{*} \mathcal{M}_{4 k+3}^{X} .
$$

We use this equation and Lemma 3.7 to complete the proof of the first two assertions for $k \geqslant 1$ by computing:

$$
(\ell / 2)^{k+1} \leqslant\left|\eta^{*}\left(\mathcal{M}_{4 k+1}^{X}(\ell)\right)\right| \leqslant\left|\eta^{*}\left(\mathcal{M}_{4 k+3}^{X}(\ell)\right)\right|
$$


Homology, Homotopy and Applications, vol. 8(2), 2006

We can now complete the proof of the main Theorem. Let

$$
\psi=-\left(\rho_{0}-\rho_{-1}\right)\left(\rho_{0}-\rho_{1}\right)=\left(\rho_{0}-\rho_{1}\right)^{2} \rho_{-1} \in R U_{0}\left(\mathbb{Z}_{\ell}\right)^{2} .
$$

We define $W^{4 k+5}=L^{4 k+5}(\ell ; 3,3, \ldots, 3,1,-1)$. Then

$$
\eta\left(M_{4 k+1, j}^{L}\right)(\rho)=\eta\left(M_{4 k+1,0}^{L}\right)\left(\psi^{j} \rho\right)=\eta\left(W^{4 k+5}\right)\left(\psi^{j+1} \rho\right) .
$$

Let $\mathcal{A}_{4 k+1}$ be the linear span of the manifolds $M_{4 k+1, j}^{L}$ for $0 \leqslant j \leqslant 2 k+1$. Let $\sigma\left(M_{4 k+1, j}^{L}\right):=\psi^{j+1}$. We extend $\sigma$ linearly to $\mathcal{A}_{4 k+1}$. We then have

$$
\eta(M)(\rho)=\eta\left(W^{4 k+5}\right)(\sigma(M) \rho) \forall M \in \mathcal{A}_{4 k+1} .
$$

If $[M]=0$ in $k o_{4 k+1}\left(B \mathbb{Z}_{\ell}, \xi_{1}\right)$, then $\eta(M)(\rho) \in \mathbb{Z}$ for all $\rho \in R U_{0}\left(\mathbb{Z}_{\ell}\right)$ by 2.5 so

$$
\eta\left(W^{4 k+5}\right)(\sigma(M) \rho) \in \mathbb{Z} \quad \forall \rho \in R U_{0}\left(\mathbb{Z}_{\ell}\right) .
$$

By Theorem 2.5(1), $\sigma(M) \in R U_{0}\left(\mathbb{Z}_{\ell}\right)^{2 k+3}$. Thus by Lemma 3.6, $\sigma$ induces a map

$$
\sigma: k o_{4 k+1}\left(B \mathbb{Z}_{\ell}, \xi_{1}\right) \rightarrow R U_{0}\left(\mathbb{Z}_{\ell}\right) / R U_{0}\left(\mathbb{Z}_{\ell}\right)^{2 k+3} .
$$

Suppose first that $m=4 k+1$. If $\sigma([M])=\sigma(M)=0$ then

$$
\eta(M)(\rho) \in \mathbb{Z} \quad \forall \rho \in R U_{0}\left(\mathbb{Z}_{\ell}\right) .
$$

Since we have that $\mathcal{G}_{L}(\ell ; \vec{a})(\bar{\lambda})=-\mathcal{G}_{L}(\ell ; \vec{a})(\lambda), \eta(M)\left(\rho_{0}\right)=0$. Thus we have $\eta(M)(\rho)=0$ for all $\rho \in R U\left(\mathbb{Z}_{\ell}\right)$ so by Theorem $2.4,[M]=0$ in $k o_{4 k+1}\left(\mathbb{Z}_{\ell} ; \xi_{1}\right)$. Thus $\sigma$ is injective. We prove the first assertion of the main Theorem by noticing that:

$$
c_{S p}\left(R S p_{0}\left(\mathbb{Z}_{\ell}\right)\right)=\operatorname{span}\left\{\psi^{j+1}: j \geqslant 0\right\} .
$$

Suppose next that $m=4 k-1$. Let $\mathcal{A}_{4 k-1}$ be the free group generated by $M_{4 k-1, j}^{X}$ for $0 \leqslant j \leqslant 2 k-2$. Let $Y^{4 k+3}:=L^{4 k+3}(\ell ; 1,3,3, \ldots, 3,1,-1)$. We then have

$$
\eta\left(M_{4 k-1, j}^{X}\right)(\rho)=\eta\left(M_{4 k-1,0}^{X}\right)\left(\psi^{j} \rho\right)=\eta\left(Y^{4 k+3}\right)\left(\psi^{j} \theta \rho\right) .
$$

Where $\theta=\left(\rho_{0}-\rho_{-1}\right)\left(\rho_{0}+\rho_{1}\right)$. Define $\sigma\left(M_{4 k-1, j}^{X}\right)=\psi^{j} \theta$ and extend $\sigma$ linearly to $\mathcal{A}_{4 k-1}$. We then have

$$
\eta(M)(\rho)=\eta\left(Y^{4 k+3}\right)(\sigma(M) \rho) \quad \text { for all } M \in \mathcal{A}_{4 k-1} .
$$

If $[M]=0$ in $k o_{4 k-1}\left(B \mathbb{Z}_{\ell}, \xi_{1}\right)$, then $\eta(M)(\rho)=0$ in $\mathbb{R} / \mathbb{Z}$ for all $\rho \in R U_{0}\left(\mathbb{Z}_{\ell}\right)$. Thus $\eta\left(Y^{4 k+3}\right)(\sigma(M) \rho)=0$ in $\mathbb{R} / \mathbb{Z}$ for all $\rho \in R U_{0}\left(\mathbb{Z}_{\ell}\right)$, so $\sigma(M) \in R U_{0}\left(\mathbb{Z}_{\ell}\right)^{2 k+2}$. Thus we may regard $\sigma$ a well defined map

$$
\sigma: k o_{4 k-1}\left(B \mathbb{Z}_{\ell}, \xi_{1}\right) \rightarrow R U_{0}\left(\mathbb{Z}_{\ell}\right) / R U_{0}\left(\mathbb{Z}_{\ell}\right)^{2 k+2} .
$$

If $\sigma([M])=0$, then $\eta(M)(\rho)=0$ for all $\rho \in R U_{0}\left(\mathbb{Z}_{\ell}\right)$ and by Theorem 2.4, $[M]=0$ in $k o_{4 k-1}\left(B \mathbb{Z}_{\ell}, \xi_{1}\right)$. Thus $\sigma$ is injective. The set $\mathcal{I}$ is generated by $\rho_{s}-\rho_{-s}$ and by $\psi^{j}$ for $j>0$. Since

$$
\theta=\rho_{1}-\rho_{-1}=\left(\rho_{0}+\rho_{1}\right)\left(\rho_{0}-\rho_{-1}\right),
$$

$\mathcal{I}$ is generated by $\psi^{j} \theta$ for $j \geqslant 0$. As we can work modulo $R U_{0}\left(\mathbb{Z}_{\ell}\right)^{2 k+2}$, we can 
restrict $0 \leqslant j \leqslant k+1<2 k+2$. Notice that

$$
\sigma\left(k o_{4 k-1}\left(B \mathbb{Z}_{\ell}, \xi_{1}\right)\right)+R U_{0}\left(\mathbb{Z}_{\ell}\right)^{2 k+2}=\mathcal{I}+R U_{0}\left(\mathbb{Z}_{\ell}\right)^{2 k+2} .
$$

Thus $k o_{4 k-1}\left(B \mathbb{Z}_{\ell}, \xi_{1}\right) \approx \mathcal{I} /\left(\mathcal{I} \cap R U_{0}\left(\mathbb{Z}_{\ell}\right)^{2 k+2}\right)$. This completes the proof of the Theorem.

\section{References}

[1] M. F. Atiyah, Characters and cohomology of finite groups, Inst. Hautes Études Sci. Publ. Math. 9 (1961), 23-64.

[2] M. F. Atiyah, K-theory, W. A. Benjamin, New York, 1967.

[3] M. F. Atiyah, V. K. Patodi, and I. M. Singer, Spectral asymmetry and Riemannian geometry I, Math. Proc. Cambridge Philos. Soc. 77 (1975), 43-69.

[4] E. Barrera-Yanez, The eta invariant, equivariant bordism, connective $K$ theory and manifolds with positive scalar curvature, Ph.D. Thesis, University of Oregon, June, 1997.

[5] E. Barrera-Yanez, and P. Gilkey, The eta invariant and the connective $K$ theory of the classifying space for the cyclic 2 groups, Annals of Global Analysis and Geometry 17 (1999), 288-299.

[6] B. Botvinnik, P. Gilkey, The Gromov-Lawson-Rosenberg conjecture: the twisted case, Houston Math. J. 23 (1997), 143-160.

[7] B. Botvinnik, P. Gilkey and S. Stolz, The Gromov-Lawson-Rosenberg conjecture for groups with periodic cohomology, J. Diff. Geo. 46 (1997), 374-405.

[8] H. Donnelly, Eta invariants for G-spaces, Indiana Univ. Math. J. 27 (1978), 889-918.

[9] P. Gilkey, The Geometry of Spherical Space Form Groups, World Scientific Press (Singapore), Series in Pure Mathematics, Vol. 7, 1989.

[10] P. Gilkey and M. Karoubi, K-theory for spherical space forms, Topology and its Applications 25 (1987), 179-184.

[11] M. Kreck and S. Stolz, $\mathbb{H}^{2}$-bundles and elliptic homology, Acta Math. 171 (1993), 231-261.

[12] S. Stolz, Simply connected manifolds with positive scalar curvature, Ann. of Math. 136 (1992), 511-540.

[13] S. Stolz, Splitting certain MSpin module spectra, Topology 33 (1994), 159180 .

Egidio Barrera-Yanez

Instituto de Matematicas, UNAM

U. Cuernavaca, Av. Universidad s/n Col. Chailpa

Cuernavaca Mor. Mexico, CP 62210

This article is available at http://intlpress.com/HHA/v8/n2/a6 